\title{
Chromatic Polynomials of Oriented Graphs
}

\author{
Danielle Cox* \\ Department of Mathematics \\ Mount Saint Vincent University \\ Halifax, Canada \\ danielle.cox@msvu.ca
}

\author{
Christopher Duffy \\ Department of Mathematics and Statistics \\ University of Saskatchewan \\ Saskatoon, Canada \\ christopher.duffy@usask.ca
}

Submitted: Oct 13, 2018; Accepted: Aug 3, 2019; Published: Sep 27, 2019

(c) The authors. Released under the CC BY-ND license (International 4.0).

\begin{abstract}
The oriented chromatic polynomial of a oriented graph outputs the number of oriented $k$-colourings for any input $k$. We fully classify those oriented graphs for which the oriented graph has the same chromatic polynomial as the underlying simple graph, closing an open problem posed by Sopena. We find that such oriented graphs can be both identified and constructed in polynomial time as they are exactly the family of quasi-transitive oriented co-interval graphs. We study the analytic properties of this polynomial and show that there exist oriented graphs which have chromatic polynomials have roots, including negative real roots, that cannot be realized as the root of any chromatic polynomial of a simple graph.
\end{abstract}

Mathematics Subject Classifications: 05C15,05C20

\section{Introduction}

An oriented graph arises by assigning directions to the edges of a simple graph. For an oriented graph, $G$, we let $U(G)$ denote the underlying simple graph. We say that $G$ is an orientation of $U(G)$. Alternately, an oriented graph is an irreflexive and anti-symmetric digraph.

The generalization of proper colouring to graph homomorphism provides a path to define proper colouring for oriented graphs in a way that takes into account the orientation. For an oriented graph $G$, an oriented $k$-colouring of $G$ is a homomorphism to a tournament (i.e., an orientation of a complete graph) on $k$ vertices. One can see that this definition of oriented colouring is equivalent the following one, which dispenses with the need to invoke homomorphism. For an oriented graph $G=\left(V_{G}, A_{G}\right)$, a function $c: V_{G} \rightarrow\{1,2, \ldots k\}$ is an oriented $k$-colouring when

\footnotetext{
${ }^{*}$ Both authors acknowledge the support of the Natural Sciences and Engineering Research Council of Canada (NSERC).
} 


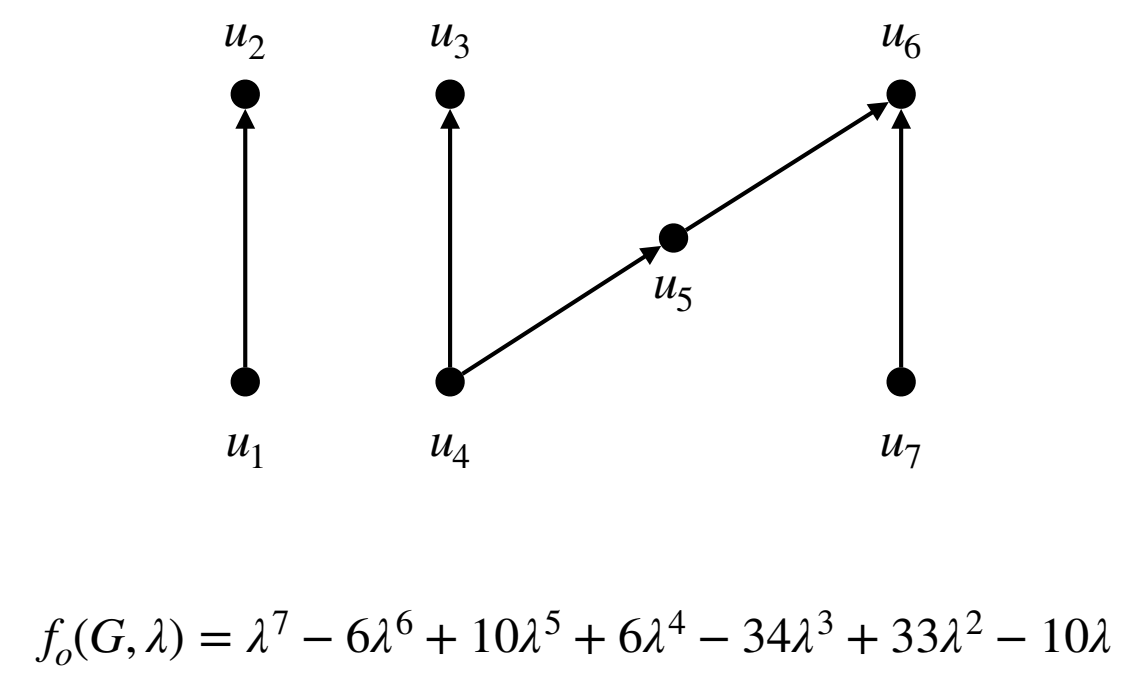

Figure 1: An oriented graph and its oriented chromatic polynomial.

1. $c(u) \neq c(v)$ for all $u v \in A_{G}$, and

2. for $u v, x y \in A_{G}$, if $c(u)=c(y)$, then $c(v) \neq c(x)$.

This second condition implies directly that non-adjacent vertices at the end of a directed path of length two (a 2-dipath) are assigned distinct colours in any oriented colouring.

Since their introduction by Courcelle in his treatment of monadic second order logic and graph structure [8], oriented colourings have provided a fertile area for fundamental research in mathematics and theoretical computer science. Many of the questions that have interested both applied and theoretical researchers in the study of graph colourings find an analogue in the study of oriented graphs. In addition to bounds for a variety of graph families $[7,9,10,11,16]$, researchers have examined the computational complexity of related decision problems [2, 13], the notion of clique for oriented graphs [3], oriented arc-colourings [15], oriented list-colourings [23] and even an oriented colouring game [14]. An excellent overview of the state-of-the-art is given in [21].

For an oriented graph $G$, we define the oriented chromatic polynomial to be the unique interpolating polynomial $f_{o}(G, \lambda)$ so that $f_{o}(G, k)$ is the number of $k$-colourings of $G$. Figure 1 gives an oriented graph together with its oriented chromatic polynomial.

The oriented chromatic polynomial was first introduced by Sopena in [20]. Here he established some basic properties of $f_{o}(G, k)$, provided a recursive construction, and exhibited some oriented graphs for which the analytic behaviour of the oriented chromatic polynomial diverged wildly from possible behaviour of the chromatic polynomial of any graph.

The definition of the oriented chromatic polynomial follows the from definition of the chromatic polynomial, $f(\Gamma, \lambda)$, for a graph $\Gamma$. The polynomial $f(\Gamma, \lambda)$ is defined to be the unique interpolating polynomial so that $f(\Gamma, k)$ is the number of $k$-colourings of $\Gamma$. 
We call an oriented graph chromatically invariant when $f_{o}(G, \lambda)=f(U(G), \lambda)$. One can see that the oriented graph given in Figure 1 is not chromatically invariant; the coefficients of $f_{o}(G, \lambda)$ do not alternate sign, a feature of every chromatic polynomial. This further implies, in fact, that there is no graph $\Gamma$ such that $f_{o}(G, \lambda)=f(\Gamma, \lambda)$. We say that a graph $\Phi$ and an oriented graph $H$ are chromatically equivalent if $f_{o}(H, \lambda)=f(\Phi, \lambda)$.

The zeros of graph polynomials have been an active area of research for many years. One such graph polynomial whose zeroes have been studied is the chromatic polynomial. The real roots of such polynomials are dense in the interval $[32 / 27, \infty)$ and there are no real roots in the interval $(0,1) \cup(1,32 / 27][4,5]$. The complex roots of such polynomials are dense in the complex plane [19].

Our work proceeds as follows. In the following section we review and recontextualize the recursive construction of $f_{o}(G, k)$ given by Sopena for the purposes of providing an explicit formula for the coefficient of $\lambda^{n-2}$ in $f_{o}(G, k)$. In Section 3 we provide a full classification of chromatically invariant oriented graphs, show they can be recognized in polynomial time and further explore the relationship between the oriented chromatic polynomial and the chromatic polynomial. We provide a partial answer to the more general question of finding chromatically equivalent pairs of oriented graphs and graphs. In Section 4 we study the roots of oriented chromatic polynomials and show that there exist oriented graphs whose oriented chromatic polynomials have roots that cannot be realized as a root of a chromatic polynomial. In particular we exhibit oriented graphs whose polynomials have negative real roots - a feature of no chromatic polynomial.

All graphs considered herein are simple. That is, we do not allow loops or multiple edges. Further, in graphs with more than one type of adjacency, we allow at most one type of adjacency between a pair of vertices. We refer the reader to [6] for graph theoretic definitions and notation.

\section{The Oriented Chromatic Polynomial}

Let $G=(V, E, A)$ be a mixed graph. That is, $G$ is a graph in which a subset (possibly empty) of the edges have been oriented to be arcs. We say that $c$ is an oriented colouring of $G$ when $c$ is an oriented colouring when $G$ is restricted to the arcs and a proper colouring when $G$ is restricted to the edges. Notice that if every pair of vertices is either adjacent or at the ends of a directed path of length 2 , then every vertex must receive a distinct colour in every colouring. We define the oriented chromatic polynomial of a mixed graph analogously to that of oriented graphs. In this section we observe that the oriented chromatic polynomial introduced by Sopena can be generalized as the oriented chromatic polynomial of mixed graphs. This generalization allows us to find a closed form for the third coefficient of the oriented chromatic polynomial of mixed graphs. As every oriented graph is a mixed graph with an empty arc set, this expression leads us to a closed form for the third coefficient of the chromatic polynomial of an oriented graph. The expression for the third coefficient provides us with a tool to study chromatically invariant oriented graphs.

We begin by providing a recursive formula for the oriented chromatic polynomial of 


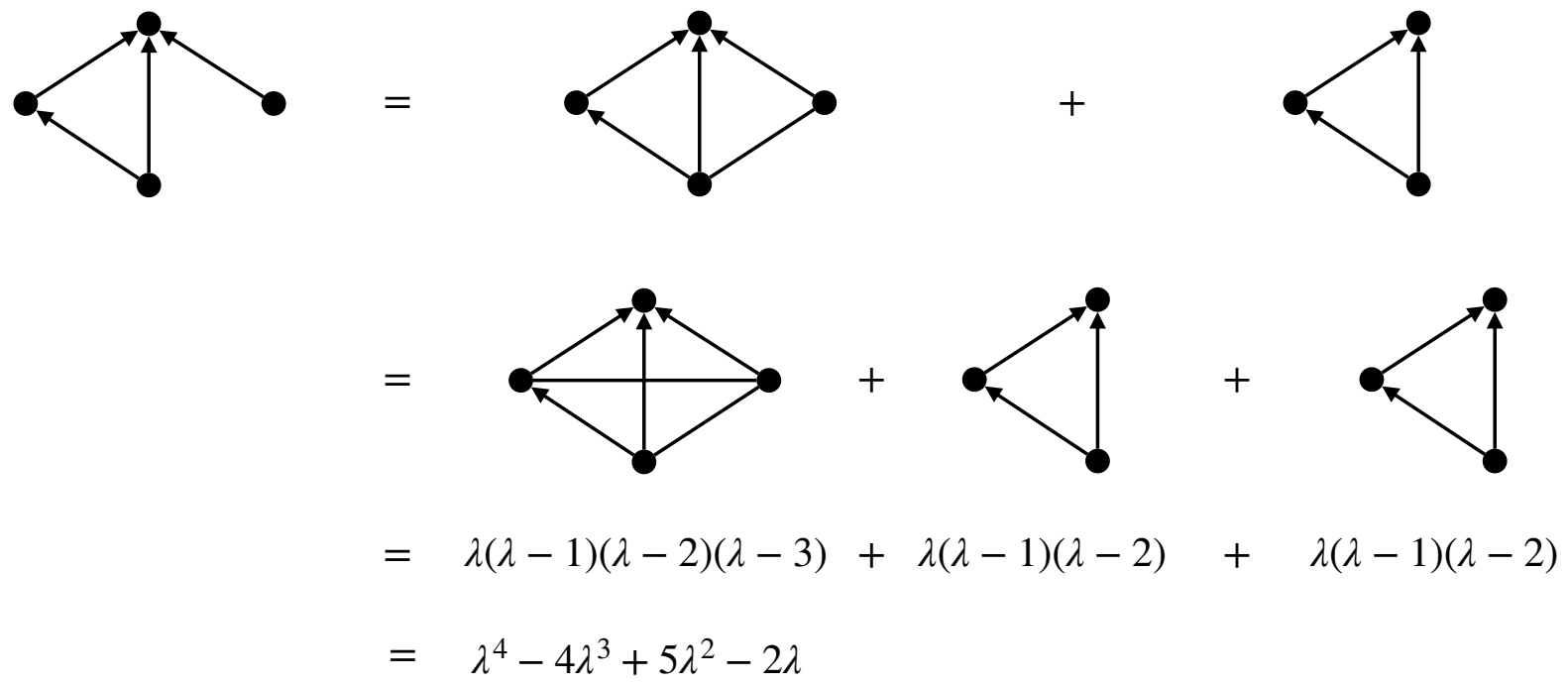

Figure 2: Computing the oriented chromatic polynomial by way of reduction.

mixed graphs. Let $G=\left(V_{G}, A_{G}, E_{G}\right)$ be a mixed graph. If every pair of vertices is either adjacent or at the ends of a directed path of length 2 (a 2-dipath), then

$$
f_{o}(G, \lambda)=\prod_{i=0}^{i=n-1}(\lambda-i)
$$

Otherwise, let $u$ and $v$ be a pair of vertices that are neither adjacent nor at the ends of a directed path of length two. In this case we have

$$
f_{o}(G, \lambda)=f_{o}(G+u v, \lambda)+f_{o}\left(G_{u v}, \lambda\right),
$$

where

- $G+u v$ is the mixed graph formed from $G$ by adding an edge between $u$ and $v$; and

- $G_{u v}$ is the mixed graph formed from $G$ by identifying $u$ and $v$ into a single vertex, deleting all parallel arcs and edges, and deleting any edge that is parallel with an arc.

Following the usual convention of having the picture of a graph stand in for its polynomial, an example of this recursion is provided in Figure 2.

The correctness of this reduction follows from the proof of the reduction provided in [20]. The appendix gives Maple code for generating the oriented chromatic polynomial of a mixed graph.

For a mixed graph $G$, let $\mathcal{D}_{G}$ be the set of pairs of vertices that are at the ends of an induced 2-dipath. In Figure 1 we have $\mathcal{D}_{G}=\left\{\left\{u_{4}, u_{6}\right\}\right\}$.

Theorem 1. [20] For any mixed graph $G=\left(V_{G}, E_{G}, A_{G}\right)$ with $n$ vertices 
1. $f(G, \lambda)$ is a polynomial of order $n$ in $\lambda$;

2. the coefficient of $\lambda^{n}$ is 1 ;

3. $f(G, \lambda)$ has no constant term;

4. the coefficient of $\lambda^{n-1}$ is $-\left(\left|A_{G}\right|+\left|E_{G}\right|+\left|\mathcal{D}_{G}\right|\right)$; and

5. if $G$ has an isolated vertex $x$, then $f_{o}(G)=\lambda \cdot f_{o}(G-x, \lambda)$.

Let $G=\left(V_{G}, A_{G}, E_{G}\right)$ be an mixed graph with non-incident arcs $u v, x y \in A_{G}$. We say that the arcs $u v, x y$ are obstructing, when

1. $u$ and $y$ are not the ends of a 2-dipath;

2. $v$ and $x$ are not the ends of a 2-dipath and

3. $u y, v x \notin E_{G}$.

Let $\mathcal{O}_{G}$ denote the set of unordered pairs of obstructing arcs in $G$, a mixed graph. In Figure 1 we have

$$
\mathcal{O}_{G}=\left\{\left\{u_{1} u_{2}, u_{4} u_{3}\right\},\left\{u_{1} u_{2}, u_{4} u_{5}\right\},\left\{u_{1} u_{2}, u_{5} u_{6}\right\},\left\{u_{1} u_{2}, u_{7} u_{6}\right\}\right\} .
$$

Lemma 2. If $\mathcal{O}_{G} \neq \emptyset$ or $\mathcal{D}_{G} \neq \emptyset$, then $f_{o}(G, \lambda) \neq f(U(G), \lambda)$.

Proof. Observe that every colouring of $G$ using $k$ colours is a colouring of $U(G)$. However if $G$ has an induced 2-dipath or a pair of obstructing arcs, then the converse does not hold. As such, there exists $k$ such that $f_{o}(G, k)<f(U(G), k)$.

Let $c_{i}(f, \lambda)$ be the coefficient of $\lambda^{n-i}$ in $f(G, \lambda)$.

Theorem 3. For a mixed graph $G=\left(V_{G}, A_{G}, E_{G}\right)$, we have

$$
c_{2}(G, \lambda)=\left(\begin{array}{c}
\left|A_{G}\right|+\left|\mathcal{D}_{D}\right|+\left|E_{G}\right| \\
2
\end{array}\right)-\left|T_{G}\right|-\left|\mathcal{D}_{G}\right|-\left|\mathcal{O}_{G}\right|,
$$

where $T_{G}$ is the set of induced subgraphs isomorphic to $K_{3}$ in $U(G)$.

Proof. Let $G$ be a minimum counter-example with respect to number of vertices. Among all such counter examples, let $G$ be the one that maximizes $\left|A_{G}\right|+\left|E_{G}\right|+\left|\mathcal{D}_{G}\right|$. Note that we may further assume $\mathcal{D}_{G}=\emptyset$ by adding an edge between every pair of vertices in $\mathcal{D}_{G}$. The resulting mixed graph has the same set of oriented colourings, and thus the same oriented chromatic polynomial.

The oriented chromatic polynomial of a mixed complete graph on $n$ is equal to the chromatic polynomial of a complete graph on $n$ vertices. The third coefficient of such a graph is given by $\left(\begin{array}{c}n \\ 3 \\ 2\end{array}\right)-\left(\begin{array}{l}n \\ 3\end{array}\right)$ [18]. Therefore the claim holds for mixed complete graphs. As $G$ is a minimum counter example, $G$ is not a complete mixed graph. As such there exists $u, v \in V(G)$ such that $u$ and $v$ are not adjacent, nor at the ends of a 2-dipath. 
Therefore $c_{2}(G, \lambda)=c_{2}(G+u v, \lambda)+c_{1}\left(G_{u v}, \lambda\right)$. By the choice of $G$, the claim holds for both $G+u v$ and $G_{u v}$.

Let $C$ be the set of common neighbours of $u$ and $v$ in $U(G)$. Each of these common neighbours forms a triangle in $G+u v$. And so $\left|T_{G}\right|=\left|T_{G+u v}\right|+|C|$. Further observe that in $G_{u v}$, the arcs/edges from $u$ and $v$ to a common neighbour $c \in C$ becomes a single adjacency in $G_{u v}$. Therefore $\left|A_{G}\right|+\left|E_{G}\right|=\left|A_{G_{u v}}\right|+\left|E_{G_{u v}}\right|-|C|$.

A pair of obstructing arcs in $G$ is not obstructing in $G+u v$ if and only if $u$ and $v$ are the head and tail, in some order, of the pair of obstructing arcs. Let $\mathcal{O}_{G}^{u v}$ be the set of such arcs. No new obstructing arcs can be created by adding an edge. Therefore $\left|\mathcal{O}_{G}\right|-\left|\mathcal{O}_{G}^{u v}\right|$ $=\left|\mathcal{O}_{G+u v}\right|$. Also note that a pair of obstructing arcs in $\left|\mathcal{O}_{G}^{u v}\right|$ form a 2-dipath in $G_{u v}$ with centre vertex $u v$. All other induced 2-dipaths in $G$ are retained in $G_{u v}$, as $u$ and $v$ are not the ends of a 2-dipath. Therefore $\left|D_{G}\right|+\left|\mathcal{O}_{G}^{u v}\right|=\left|D_{G_{u v}}\right|$.

$$
\begin{gathered}
c_{2}(G, \lambda)=c_{2}(G+u v, \lambda)+c_{1}\left(G_{u v}, \lambda\right) \\
=\left(\begin{array}{c}
\left|A_{G}\right|+\left|D_{G}\right|+\left|E_{G}\right|+1 \\
2
\end{array}\right)-\left(\left|T_{G+u v}\right|+\left|D_{G+u v}\right|+\left|\mathcal{O}_{G+u v}\right|\right) \\
-\left(\left|A_{G_{u v}}\right|+\left|E_{G_{u v}}\right|+\left|D_{G_{u v}}\right|\right) \\
=\left(\begin{array}{c}
\left|A_{G}\right|+\left|D_{G}\right|+\left|E_{G}\right|+1 \\
2
\end{array}\right)-\left(\left|T_{G}\right|-|C|+\left|D_{G}\right|+\left|\mathcal{O}_{G}\right|-\left|\mathcal{O}_{G}^{u v}\right|\right) \\
-\left(\left|A_{G}\right|+\left|E_{G}\right|+|C|+\left|D_{G}\right|+\left|\mathcal{O}_{G}^{u v}\right|\right) \\
=\left(\begin{array}{c}
\left|A_{G}\right|+\left|D_{G}\right|+\left|E_{G}\right|+1 \\
\left.2 \quad \begin{array}{c}
\left|A_{G}\right|+\left|D_{G}\right|+\left|E_{G}\right| \\
2
\end{array}\right)-\left|T_{G}\right|-\left|D_{G}\right|-\left|\mathcal{O}_{G} \cdot\right|
\end{array}\right.
\end{gathered}
$$

Thus the claim holds for $G$, contradicting the choice of $G$ as a minimum counter example.

For the case $E=\emptyset$, we arrive at the desired result for oriented graphs.

Corollary 4. For an oriented graph $G$, we have

$$
c_{2}(G, \lambda)=\left(\begin{array}{c}
\left|A_{G}\right|+\left|\mathcal{D}_{G}\right| \\
2
\end{array}\right)-\left|T_{G}\right|-\left|\mathcal{D}_{G}\right|-\left|\mathcal{O}_{G}\right| .
$$

We further note that in the case $A=\emptyset$, we arrive at the usual result for the third coefficient of the chromatic polynomial: $c_{2}(G, \lambda)=\left(\begin{array}{c}\left|E_{G}\right| \\ 2\end{array}\right)-\left|T_{G}\right|$. 


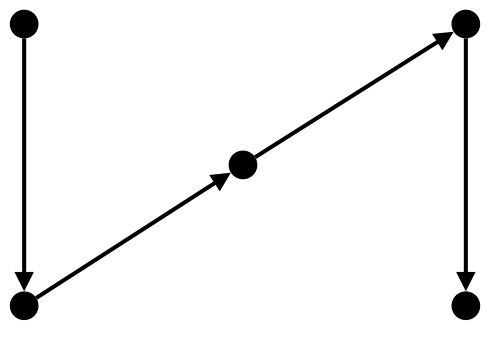

$G$

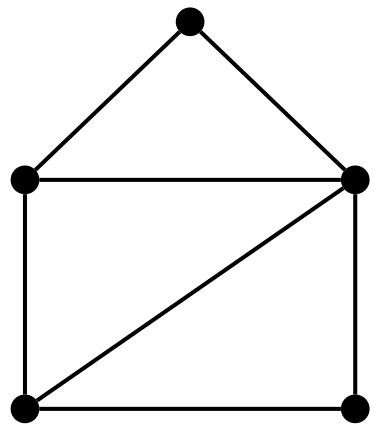

$\Gamma$

$$
f_{o}(G, \lambda)=f(\Gamma, \lambda)=\lambda^{5}-7 \lambda^{4}+18 \lambda^{3}-20 \lambda^{2}+8 \lambda
$$

Figure 3: An oriented graph and a graph with the same chromatic polynomial.

\section{Oriented Chromatic Equivalence}

A folklore construction gives an orientation $G$ of $K_{n, n}$ so that the resulting oriented graph has chromatic number $2 n$ (see [9]). This common example is used to convince the reader that the oriented chromatic number and the chromatic number of the underlying simple graph can be arbitrarily far apart. We note, however that the set of colourings of $G$ using $\lambda \geqslant 2 n$ colours is exactly that of colourings of $K_{2 n}$ using $\lambda$ colours. And so though the chromatic number of $U(G)$ differs greatly to $G$, there is still a relationship between colourings of $G$ and colourings of some simple graph. In this section we find a set of sufficient conditions so that the $\lambda$-colourings of an oriented graph $G$ are exactly those of some simple graph $\Gamma$. We conclude this section by using these sufficient conditions to compute the chromatic polynomial of orientations of stars.

We are interested in the following decision problems:

CHROM-INVAR

Instance: A graph $\Gamma$.

Question: Is there an orientation $O(\Gamma)$ such that $f_{o}(O(\Gamma), \lambda)=f(\Gamma, \lambda)$ ?

OCHROM-INVAR

Instance: An oriented graph $G$.

Question: Does $f_{o}(G, \lambda)=f(U(G), \lambda)$ ?

OCHROM-EQUIV

Instance: An oriented graph $G$.

Question: Is there a graph $\Gamma$ such that $f_{o}(G, \lambda)=f(\Gamma, \lambda)$ ?

Figure 3 gives an example of an oriented graph $G$ and a graph $\Gamma$ so that $G$ and $\Gamma$ are chromatically equivalent.

Let $G$ be an oriented graph. Let $G^{\star}$ be the mixed graph resulting from $G$ by adding 
an edge between $u$ and $v$ whenever $u$ and $v$ are at the ends of an induced 2-dipath. We observe the following.

Lemma 5. $f_{o}(G, \lambda)=f_{o}\left(G^{\star}, \lambda\right)$.

Proof. $G$ is a subgraph of $G^{\star}$. Therefore $f_{o}(G, \lambda) \leqslant f_{o}\left(G^{\star}, \lambda\right)$. Every oriented colouring of $G^{\star}$ using $k$ colours is also an oriented colouring of $G$, therefore $f_{o}(G, \lambda) \geqslant f_{o}\left(G^{\star}, \lambda\right)$.

Theorem 6. For $G$, an oriented graph, $f_{o}(G, \lambda)=f\left(U\left(G^{\star}\right), \lambda\right)$ if and only if $\mathcal{O}_{G}=\emptyset$.

Proof. By Lemma 5 it suffices to show $f_{o}\left(G^{\star}, \lambda\right)=f\left(U\left(G^{\star}\right), \lambda\right)$. Notice that $G$ has no obstructing arcs if and only if $G^{\star}$ has no obstructing arcs. By Lemma 2 it suffices to show that if $G^{\star}$ has no obstructing arcs, then every colouring of $U\left(G^{\star}\right)$ is an oriented colouring of $G$. Let $c$ be a colouring of $U\left(G^{\star}\right)$. Since $c$ is a proper colouring, if $c$ is not an oriented colouring of $G^{\star}$, then the second condition of oriented colouring as been violated. However, this not possible as $G^{\star}$ has neither an induced 2-dipath nor a pair of obstructing arcs.

Recall the result of Corollary 4. If $G$ has no pair of obstructing arcs, then in $G^{\star}$ we have $\mathcal{O}_{G}=\mathcal{D}_{G}=\emptyset$. And so $c_{2}\left(G^{\star}, \lambda\right)=\left(\begin{array}{c}\left|A_{G}\right|+\left|E_{G}\right| \\ 2\end{array}\right)-\left|T_{G}\right|$. Notice that this is exactly the third coefficient of the chromatic polynomial of $U\left(G^{\star}\right)$.

Corollary 7. An oriented graph $G$ is chromatically invariant if and only if $G$ has no induced 2-dipath and $U(G)$ is $2 K_{2}$-free.

Proof. Assume $G$ has no induced 2-dipath and that $U(G)$ is $2 K_{2}$-free. It follows directly that $G$ has no obstructing arcs and that $G=G^{\star}$. The conclusion follows by Theorem 6 .

Let $G$ be a chromatically invariant oriented graph. By definition, every proper $k$ colouring of $U(G)$ is an oriented colouring of $G$. Therefore $G$ has no induced 2-dipath, nor does $G$ contain a pair of obstructing arcs. Since $G$ contains no pair of obstructing arcs, if $U(G)$ contains an induced copy of $2 K_{2}$, say $u v, x y$, then without loss of generality, there must be an induced 2-dipath between $u$ and $y$. This contradicts that $G$ contains no induced 2-dipath. Therefore $G$ has no induced 2-dipath and $U(G)$ is $2 K_{2}$-free.

Introduced by Ghouila-Houri, oriented graphs that contain no induced 2-dipath are called quasi-transitive.

Theorem 8. [12] A graph $\Gamma$ admits a quasi-transitive orientation if and only if $\Gamma$ is a comparability graph.

Notice that the family of $2 K_{2}$-free comparability graphs is exactly the family of cointerval graphs. And so combining Corollary 7 and Theorem 8 yields the following classification.

Theorem 9. An oriented graph is chromatically invariant if and only if it is a quasitransitive orientation of a co-interval graph. 
This theorem fully classifies chromatically invariant oriented graphs, as well as those graphs that admit a chromatically invariant orientation. This closes an open problem posed by Sopena in [20] and provides a geometric interpretation of chromatically invariant oriented graphs as co-interval graphs.

Theorem 9 implies that the decision problems CHROM-INVAR and OCHROM-INVAR can be restated in terms of co-interval graph recognition. As co-interval graphs can be identified in linear time [22], we arrive at the following classification of CHROM-INVAR and OCHROM-INVAR.

Corollary 10. The decision problems CHROM-INVAR and OCHROM-INVAR are Polynomial.

We note that such an orientation of a co-interval graph need not be unique (up to converse). There are many methods in the literature (for example see [1]) that give a quasitransitive orientation of a comparability graph. A common element of these methods is the construction of an auxiliary graph, $\operatorname{Aux}(G)$, so that a 2-colouring of $G$ corresponds to a quasi-transitive orientation. Such constructions imply that a comparability graph has a unique quasi-transitive orientation (up to converse) if and only if $\operatorname{Aux}(G)$ is connected. As such constructions can be carried out in polynomial time, we find that given a cointerval graph $\Gamma$, one may find in polynomial time an orientation of $\Gamma, O(\Gamma)$, so that $f(\Gamma, \lambda)=f_{o}(O(\Gamma), \lambda)$.

We now consider an application of Theorem 6 and find the oriented chromatic polynomial of the family of orientations of stars. Let $S_{i, o}$ be the orientation of a star on $i+o+1$ vertices, with centre vertex $x$, so that $x$ has $i$ in-neighbours and $o$ out-neighbours.

Corollary 11. $f_{o}\left(S_{i, o}\right)=\lambda \cdot f\left(K_{i, o}, \lambda-1\right)$

Proof. Observe that $S_{i, o}^{\star}$ has no obstructing arcs. Further observe that $U\left(S_{i, o}^{\star}\right)$ consists of a copy of $K_{i, o}$ together with a universal vertex. By Theorem 6 , we have $f_{o}\left(S_{i, o}\right)=$ $\lambda \cdot f\left(K_{i, o}, \lambda-1\right)$.

Conversely, using the results from Section 2, one can find families of oriented graphs for which there is no chromatically equivalent graph. Let $G$ be an orientation of $t K_{2}$ for some $t>1$. Recalling the notation of the previous section we have

- $\left|A_{G}\right|=t$

- $\left|E_{G}\right|=\left|\mathcal{D}_{G}\right|=0$;

- $\left|\mathcal{O}_{G}\right|=\left(\begin{array}{l}t \\ 2\end{array}\right)$; and

- $\left|T_{G}\right|=0$.

If there exists $\Gamma$ such that $f_{o}(G, \lambda)=f(\Gamma, \lambda)$, then by Theorem 1 and Corollary 4 , it must be that $\Gamma$ has $2 t$ vertices, $t$ edges and $\left(\begin{array}{l}t \\ 2\end{array}\right)$ copies of $K_{3}$. A simple counting argument implies that no such $\Gamma$ can exist. 
Conjecture 12. Let $G$ be an oriented graph. There is a graph $\Gamma$ such that $G$ and $\Gamma$ are chromatically equivalent if and only if $G$ has no obstructing pairs of arcs.

When restricted to oriented graphs that contain no pair of obstructing arcs, OCHROMEQUIV is Polynomial; every instance is a YES instance. However, for arbitrary inputs is not clear if OCHROM-EQUIV is contained in NP, as constructing the chromatic polynomial of a graph is NP-hard. We conjecture, however, that those oriented graphs with no obstructing arcs are the only oriented graphs whose $\lambda$-colourings have a one-to-one correspondence with the $\lambda$-colourings of some graph $\Gamma$. If Conjecture 12 is true, then OCHROM-EQUIV is Polynomial for arbitrary inputs.

Conjecture 12 is not true when we allow $G$ to be a mixed graph, even when we require $A \neq \emptyset$. Let $H$ be the mixed graph formed from a pair of disjoint arcs by adding an edge between the heads and an edge between the tails. Using the reduction given in Section 2, we find $f_{o}(H, \lambda)=\lambda^{4}-4 \lambda^{3}+5 \lambda^{2}-2 \lambda$. Notably, this is exactly the chromatic polynomial of the example, $G$, given in Figure 2. The oriented graph $G$ has $\mathcal{D}_{G}=\emptyset$. Therefore $G=G^{\star}$. Further $\mathcal{O}_{G}=\emptyset$. And so by Theorem 6 we have $f(U(G), \lambda)=f_{o}(G, \lambda)=$ $f_{o}(H, \lambda)$. In particular, $H$ has a pair of obstructing arcs, but yet there is graph $\Gamma$ such that $f_{o}(H, \lambda)=f(\Gamma, \lambda)$. From this example, one may generate an example on $n$ vertices for any $n \geqslant 4$ by repeatedly adding universal vertices to $H$ and $U(G)$.

\section{Roots of Oriented Chromatic Polynomials}

The location of the roots of polynomials has been well studied for a variety of graph polynomials, such the independence, domination, reliability and chromatic polynomials. In this section we provide results regarding the roots of the oriented chromatic polynomial. Chromatic polynomials have roots that are dense in the complex plane [19]. Their coefficients alternate in sign and hence have no negative real roots [17]. We show the following:

Theorem 13. For every integer $k>0$, there exists an oriented graph $G$ so that $f_{o}(G, \lambda)$ has a root $k^{\prime}$ so that $k^{\prime}<-k$.

Proof. Let $D_{n}$ be the oriented graph on $n$ vertices that consists of a directed path $v_{1}, v_{2}, v_{3}, v_{4}$ with $n-4$ leaves, $x$ directed from $x$ to $v_{4}$.

The oriented chromatic polynomial can be computed in the following way. Vertex $v_{1}$ has $\lambda$ colour choices, $v_{2}$ has $\lambda-1$ colour choices and $v_{3}$ has $\lambda-2$ colour choices. Now for $v_{4}$ we have two options. If $v_{4}$ is the same colour as $v_{1}$ then the $n-4$ leaves have $\lambda-2$ choices of colour, since it can not be the same colour at $v_{4}$ or $v_{2}$. If $v_{4}$ is a different colour than the others in the path it has $\lambda-3$ colour choices and the leaves have $\lambda-1$ colour choices. This means

$$
f_{o}\left(D_{n}, \lambda\right)=\lambda(\lambda-1)(\lambda-2)\left((\lambda-2)^{n-4}+(\lambda-3)(\lambda-1)^{n-4}\right)
$$

The polynomial $f_{o}\left(D_{n}, \lambda\right)$ has real roots at $\lambda=0,1,2$. We show we can obtain arbitrarily large negative real roots by showing that a real root exists between $\lambda=-n$ and $\lambda=-\ln (n)$ for $n$ even. 
Observe,

$f_{o}\left(D_{n},-n\right)=(-1)^{3} n(n+1)(n+2)\left((-1)^{n-4}(n+2)^{n-4}+(-1)^{n-3}(n+3)(n+1)^{n-4}\right)$

It can be shown that $(n+2)^{n-4}<(n+3)(n+1)^{n-4}$ if

$$
\left(1+\frac{1}{n+1}\right)^{n-4}<n+3
$$

The quantity $\left(1+\frac{1}{n+1}\right)^{n-4}$ is bounded above by $e$, and $e<n+3$ for all values of $n$, therefore $f_{o}\left(D_{n},-n\right)>0$ for all even values of $n$.

Now consider $f_{o}\left(D_{n},-\ln (n)\right)$.

$$
\begin{aligned}
f_{o}\left(D_{n},-\ln (n)\right)= & (-1)^{3}(\ln (n))(\ln (n)+1)(\ln (n)+2) * \\
& \left((-1)^{n-4}(\ln (n)+2)^{n-4}+(-1)^{n-3}(\ln (n)+3)(\ln (n)+1)^{n-4}\right)
\end{aligned}
$$

Clearly $(\ln (n)+2)^{n-4}>(\ln (n)+3)(\ln (n)+1)^{n-4}$ when

$$
\left(1+\frac{1}{\ln (n)+1}\right)^{n-4}>\ln (n)+3 .
$$

Let $g(n)=\left(1+\frac{1}{\ln (n)+1}\right)^{n-4}-\ln (n)-3$. The derivative of this function is $g^{\prime}(n)=\left(1+(\ln (n)+1)^{-1}\right)^{n-4}\left(\ln \left(1+(\ln (n)+1)^{-1}\right)-\frac{n-4}{(\ln (n)+1)^{2} n\left(1+(\ln (n)+1)^{-1}\right)}\right)-\frac{1}{n}$.

We have $\lim _{n \rightarrow \infty} g^{\prime}(n)=\infty$, thus there exists $N$ so that for all $n>N$. Further, $g(n)$ is an increasing function, as the derivative of $g$ is positive, and hence $g(n)>0$ and $f_{o}\left(D_{n},-\ln (n)\right)<0$ for large values of $n$.

It then follows by the intermediate value theorem that $f_{o}\left(D_{n}, \lambda\right)$ can have an arbitrarily large negative root.

A chromatic polynomial cannot have root in the interval $(-\infty, 0) \cup(0,1) \cup\left(1, \frac{32}{27}\right)[17]$. We have shown that oriented chromatic polynomials can have negative real roots. In addition, there exist oriented chromatic polynomials in the interval $(0,1)$, as $f_{o}\left(D_{5}, \lambda\right)$ has a root at $\lambda=\frac{3}{2}-\frac{\sqrt{5}}{2}$. Open problems regarding the roots of oriented chromatic polynomials include: does there exist an oriented graph whose real roots lie in $\left(1, \frac{32}{27}\right)$ ? What is the closure of the complex roots for the oriented chromatic polynomial?

\section{Conclusion}

The study of oriented graphs often goes hand-in-hand with that of signed graphs. Though the methods contained herein will extend to the study of chromatic polynomials of signed graphs, there will be a marked difference in the classification of chromatically invariant signed graphs. For example, letting all the edges of $2 K_{2}$ be positive leads to a chromatically invariant signed graph. However, all possible orientations of $2 K_{2}$ leads to an oriented 
graph that is not chromatically invariant. Of course, with this approach, every graph can have edge signs trivially assigned so that the resulting signed graph has the same chromatic polynomial as the underlying graph. And so one may require that there is at least one edge of each sign. With this added restriction it is unclear if chromatically invariant signed graphs can be identified in polynomial time, as we expect the characterization to require that signs be given so that there is no $2 K_{2}$ where the edges have different signs. Similarly, the generalization of signed graphs and oriented graphs to $(m, n)$-mixed graphs should yield a definition of a chromatic polynomial that obeys the reduction outlined in Section 2. Consequently we expect the results of Theorems 1 and 3 to generalize in the same manner. One may also ask, then, for which graphs $\Gamma$ is there an assignment of arcs, edges, and corresponding colours, so that the resulting $(m, n)$-mixed graph is chromatically invariant. We have shown that for oriented graphs that $\Gamma$ must be a co-interval graph.

\section{Acknowledgments}

The authors thank Gary MacGillivray for discussions regarding the recursive construction of the oriented chromatic polynomial.

\section{References}

[1] J. Bang-Jensen and G. Z. Gutin. Digraphs: theory, algorithms and applications. Springer Science \& Business Media, 2008.

[2] J. Bang-Jensen, P. Hell, and G. MacGillivray. The complexity of colouring by semicomplete graphs. SIAM Journal on Discrete Mathematics, 1(3):281-298, 1988.

[3] J. Bensmail, C. Duffy, and S. Sen. Analogues of cliques for $(m, n)$-colored mixed graphs. Graphs and Combinatorics, 33(4):735-750, 2017.

[4] B. Jackson. A zero-free interval for the chromatic polynomials of graphs. Combinatorics, Probability and Computing, 2:325-336, 1993.

[5] B. Jackson. The zero-free interval for the chromatic polynomials of graphs. Combinatorics, Probability and Computing, 6:497-506, 1997.

[6] J. Bondy and U. Murty. Graph Theory. Number 244 in Graduate Texts in Mathematics. Springer, 2008.

[7] O. Borodin, A. Kostochka, J. Nešetřil, A. Raspaud, and E. Sopena. On the maximum average degree and the oriented chromatic number of a graph. Discrete Mathematics, 206:77-89, 1999.

[8] B. Courcelle. The monadic second order logic of graphs VI: On several representations of graphs by relational structures. Discrete Applied Mathematics, 54:117-149, 1994.

[9] C. Duffy, G. MacGillivray, and E. Sopena. Oriented colourings of graphs with maximum degree three and four. Discrete Mathematics, 342:959-974, 2019. 
[10] J. Dybizbański and A. Szepietowski. The oriented chromatic number of Halin graphs. Information Processing Letters, 114(1):45-49, 2014.

[11] G. Fertin, A. Raspaud, and A. Roychowdhury. On the oriented chromatic number of grids. Inform. Proc. Letters, 85:261-266, 2003.

[12] A. Ghouila-Houri. Caractérisation des graphes non orientés dont on peut orienter les arêtes de manière à obtenir le graphe d'une relation d'ordre. C. R. Acad. Sci. Paris, 254:1370 - 1371, 1962.

[13] W. F. Klostermeyer and G. MacGillivray. Pushing vertices and oriented colorings. Bull. Inst. Combin. Appl, 40:49-58, 2004.

[14] J. Nešetril and E. Sopena. On the oriented game chromatic number. Electronic Journal of Combinatorics, 8(2):\#R14, 2001.

[15] P. Ochem, A. Pinlou, and E. Sopena. On the oriented chromatic index of oriented graphs. Journal of Graph Theory, 57(4):313-332, 2008.

[16] A. Raspaud and E. Sopena. Good and semi-strong colorings of oriented graphs. Information Processing Letters, 51:171-174, 1994.

[17] R. Read and W. Tutte. Selected Topics in Graph Theory 3, Chapter: Chromatic Polynomial. Number 244. Academic Press Ltd., 1988.

[18] R. C. Read. An introduction to chromatic polynomials. Journal of Combinatorial Theory, 4(1):52-71, 1968.

[19] A. Sokal. Chromatic roots are dense in the whole complex plane. Combinatorics, Probability and Computing, 13(2):221-261, 2004.

[20] E. Sopena. Computing chromatic polynomial of oriented graphs. Université de Bordeaux 1/CNRS. Laboratoire Bordelais de Recherche en Informatique [LaBRI], 1994.

[21] E. Sopena. Homomorphisms and colourings of oriented graphs: An updated survey. Discrete Mathematics, 339(7):1993-2005, 2016.

[22] J. P. Spinrad. Efficient graph representations. American Mathematical Society, 2003.

[23] Z. Tuza and M. Voigt. Oriented list colorings of graphs. Journal of Graph Theory, 36(4):217-229, 2001. 


\section{Appendix}

\#ocpoly recursively computes the chromatic polynomial of a mixed graph.

$\# G$ is the directed graph, $E$ is the set of edges and $s$ is an integer that is larger than the number of vertices of $G$.

\#We use s to give the right label to the newly created vertex when we identify a pair of vertices. This preserves vertex label uniqueness.

$\#$ We apply the reduction from Section 1 of the paper (see Figure 2).

\#In the base case the input is a clique and so every vertex needs to get a unique colour

ocpoly $:=\operatorname{proc}(G, E, s)$ option (remember);

local $i, A, V$, locals, $p 2$

p2:=0:

locals $:=s+1$;

$H:=\operatorname{Underlying} \operatorname{Graph}(G)$

$\operatorname{Ver}:=\operatorname{Vertices}(G)$

$V:=\{\}:$

for $l$ from 1 to nops (Ver) do

od:

if Degree $(H, o p(l, V e r)) \neq 0$ then $V:=V$ union $\{o p(l, V e r)\}$ fi

iso : $=\operatorname{nops}(\operatorname{Ver})-\operatorname{nops}(V)$;

$E H:=\operatorname{Edges}(H)$;

new $A:=\operatorname{choose}(\operatorname{convert}(V$, set $), 2)$ minus $E H$ minus $E$;

E1 :=E;

new Garcs $:=\{\}:$

newEdges $:=\{\}$

\#If all pairs of vertices are adjacent (in any sense) then it is an oriented clique and so all vertices need their own colour. In this case we return the falling factorial.

if $\operatorname{nops}(\operatorname{new} A)=0$ then

$$
p 2:=x^{i s o} \cdot\left(\prod_{i=0}^{\operatorname{nops}(V)-1}(x-i)\right)
$$

return $(p 2)$;

\#Otherwise, there is a pair of non-adjacnent vertices that can be identifed.

else

\#Find a pair of vertices a1, a2 such that a1 and a 2 can be identified.

$a:=o p(1$, new $A)$

$a 1:=o p(1, a)$

$a 2:=o p(2, a)$

$f l:=0$ :

if $\operatorname{convert}(\operatorname{Departures}(G, a 1)$, set) intersect convert $(\operatorname{Arrivals}(G, a 2)$, set $) \neq\{\}$ then $f l:=1 ; \mathbf{f i}$

if convert (Arrivals $(G, a 1)$, set) intersect convert $(\operatorname{Departures}(G, a 2)$, set $) \neq\{\}$ then $f l:=1$; fi:

if $f l=0$ then

$E G:=\operatorname{Edges}(G)$

for $i$ from 1 to $\operatorname{nops}(E G)$ do \#Update the arc set after identying the vertices.

if $o p(1, o p(i, E G))=a 1$ then newGarcs $:=$ new Garcs union $\{[s, o p(2, o p(i, E G))]\} ;$;

elif $o p(1, o p(i, E G))=a 2$ then new Garcs :=newGarcs union $\{[s, o p(2, o p(i, E G))]\}$;

elif $o p(2, o p(i, E G))=a 1$ then new Garcs $:=\operatorname{new} \operatorname{Garcs}$ union $\{[o p(1, o p(i, E G)), s]\}$;

elif $\operatorname{op}(2, \operatorname{op}(i, E G))=a 2$ then new Garcs $:=\operatorname{new} \operatorname{Garcs}$ union $\{[\operatorname{op}(1, \operatorname{op}(i, E G)), s]\}$;

else newGarcs $:=$ new Garcs union $\{\operatorname{convert}(\operatorname{op}(i, E G)$, list $)\}$;

od:

fi: \#changes $E$ to $E G$, made set a list

for $i$ from 1 to $n o p s(E 1)$ do \#Update the edge set after identifying the vertices.

if $o p(1, o p(i, E 1))=a 1$ then newEdges $:=\operatorname{newEdges}$ union $\{\{s, o p(2, o p(i, E 1))\}\}$;

elif $o p(1, o p(i, E 1))=a 2$ then newEdges $:=$ newEdges union $\{\{s, o p(2, o p(i, E 1))\}\}$;

elif $o p(2, o p(i, E 1))=a l$ then newEdges $:=$ newEdges union $\{\{o p(1, o p(i, E 1)), s\}\}$;

elif $o p(2, o p(i, E 1))=a 2$ then newEdges $:=n e w E d g e s$ union $\{\{o p(1, o p(i, E 1)), s\}\}$;

else newEdges $:=$ newEdges union $\{o p(i, E 1)\}$;

od:

new $G:=\operatorname{Digraph}($ new Garcs $)$;

p2 $:=x^{\text {iso }} \cdot($ ocpoly $($ new $G$, newEdges, locals $))$;

else $p 2=0$;

fi:

$E 1:=E 1$ union $\{\{a 1, a 2\}\}$;

$p 3:=\operatorname{ocpoly}(G, E 1$, locals $))$

fi:

return $(\operatorname{expand}((p 2+p 3)))$;

end proc: 